\title{
The 7th August 1998, Nairobi American Embassy Bomb Blasts Survivors with Orthopaedic Conditions
}

\author{
G.K. Museve, MBChB, MMed (Surg), Department of Orthopaedic Surgery, College of Health Sciences, \\ University of Nairobi, P.O. Box 29829-00200, Nairobi, Kenya \\ Email:gkmuseve@yahoo.co.uk
}

\begin{abstract}
Objective: To determine the demography, occupation, types of orthopaedic conditions and conributory factors among survivors of the bomb blast.
\end{abstract}

Design: Retrospective descriptive study.

Setting: Health facilities and rehabilitation centres at which survivors attended, Africa medical Research Foundation (AMREF) and at the author's follow up clinic. The study period of 1999 to 2002.

Results: Out of study population of $1090,315(29 \%)$ had orthopaedic conditions. Of the 305 documented cases, $64 \%$ were married and $22 \%$ were single, while $64 \%$ had between $0-3$ and $25 \%$ had $4-6$ children. Two hundred and seventy seven survivors were subsequently followed during the AMREF implemented medical assistance programme (1999-2002). Female victims were $172(62.1 \%)$ giving a female to male ratio of 1.6:1. Eighty six percent were in age group 21 to 50 years, with a range of 14 to 77 years and a mean of 36 years. Professionals, executives, managers, semi professionals and skilled workers comprised $89.5 \%$ of the victims when documented occupation was considered and was $66 \%$ when undocumented occupation was included. The survivors who were inside buildings comprised $71.8 \%$ and $75.5 \%$ were within 100 metres from the bomb blast site. Fifty percent were first treated in public hospitals. The following injuries were noted:Fractures $\mathbf{1 1 . 9 \%}$, tendon injuries $\mathbf{5 . 1 \%}$ nerve injuries $3.6 \%$ and back injuries $\mathbf{2 8 . 5} \%$. The victims who were inside buildings were $\mathbf{7 0 . 6} \%$ and those within $\mathbf{1 0 0}$ metres radius from the blast site were $\mathbf{7 4 . 3 \%}$. Survivors with joint injuries were $24.2 \%$.

Conclusion: The bomb victims were in economically productive group, and a large percentage having 0-3 dependants. Being inside a building and within 100 metres from the blast carried the largest risk of injury. $A$ re-examination of building by- laws and safety is therefore suggested as well as adequate staffing, training, and equipping of public hospitals since $50 \%$ of victims received their first treatment in public hospitals. The economic impact, when finally assessed will be considerable due to loss of property, lives, earnings and cost of medical care. The formation of a disaster management body that will draw up protocols for handling disaster is strongly recommended.

\section{INTRODUCTION}

Kenya had never experienced a suicidal terrorist bombing before, until $7^{\text {th }}$ August 1998 when it occurred at the Nairobi American Embassy in which human lives were lost and property damaged.

Terrorist activity is one of the ugly facts of modern life that has a wide distribution over the world $(1,2)$. Between 1980 and 1990 there were 12,216 intentional bombing incidences in the United States of America (2,3). Between August 1969 and June 1972, there were 1532 explosions victims treated at Royal Victoria Hospital, Belfast, Northern Ireland.
There are several mechanisms by which blasts can produce injury patterns. Primary blast injuries are produced by initial blast wave on the body, which moves concentrically from the centre point of the explosion.

Secondary blast injuries result from penetrating or non-penetrating damage caused by debris energized by the blast wind, which follows the blast wave. The blast wind is a displacement of air caused by the explosion.

Tertiary blast injuries result from whole body displacement and resultant traumatic impact with environmental objects, due to the blast wind. Fourth degree or miscellaneous blast injuries include thermal radiation and crush injuries from collapsing structures. 
Traumatic amputations are probably the most dramatic serious orthopaedic consequence of explosions (1). They carry a high risk of mortality $(1,5)$. In Hadden et al (1) study involving 1532 victims, four of the 20 victims with traumatic amputations died, in which the total death toll was nine and in the Hull et al (5) forensic study, they reported 34 deaths with 73 amputations. Hull et al concluded that amputation itself was a secondary event arising from the flow of combustion products in an already fractured $\operatorname{limb}(5,6)$.

There are not many documented studies on bomb blast injuries. Hadden et al found that females formed the majority of the victims (60\%), but among those who were admitted, the order was reversed (58\%) (1).

In Oklahoma City Bombing (7)in which 842 persons were injured, $80 \%$ of the injuries and deaths occurred among people of between 20-59 years, this being an economically active group. Sixty one per cent non-fatal injuries occurred in buildings close to each other while $25 \%$ of non-fatal injuries occurred in other buildings. Only $9 \%$ of the non- fatal injuries occurred in outdoor locations. The bombed building produced a combined non-fatal injury of $91 \%$.

Various studies have documented fracture or dislocation incidences of between $6 \%$ and $12.9 \%$ of the victims $(7,8)$. Hadden and colleagues documented fractures, dislocations and sprains in $4.1 \%$ of the 1523 survivors. The following was the distribution of the fractures: Tibia-fibula, $16.2 \%$, radius/ulna and ankle, $14.9 \%$ each, hand and tibia, $13.5 \%$ each (1). Seven patients had nerve injuries. All recovered fully except one (sciatic nerve) which only partially recovered. Sprain, strain and contusion occurred in 26\% (260 of the 790 patients (1). Severance H.W. gave suggestions on the organization and management of blast related injuries involving mass casualties. He cautioned on the possibility of second blast "come- on bomb". He gave suggestions on on-site management of blast injuries with mass casualties, protocols for in-hospital patients, in- hospital emergency/trauma centre management of blast victims (2).

Terrorist bombing has a large economic loss. In the Oklahoma bombing, the charges for acute care at hospitals exceeded 2.5 million U.S. dollars. This excluded emergency, transport, physician, surgeon and rehabilitation charges. The economic impact will be considerable if the total costs, including damage to property are considered (7).

The objective of the study was to determine the demography, occupation, types of orthopaedic conditions and contributory factors among survivors of the $7^{\text {th }}$ August 1998 bomb blast, at the Nairobi American Embassy.

The aims of the study were as follows:-

- To determine the age, and sex of the survivors.

- To determine their marital status.

- To determine occupations of the victims.

- To determine the number of dependants the survivors had.

- To determine how the location and distance from the blast affected the injuries.

- To determine which first facility the survivors attended after the injury and the conditions arising from injuries or surgery in the follow up cases.

- To determine what operations were done and the complications arising from injuries in the followed up cases.

- To give recommendations based on the study findings.

\section{MATERIALS AND METHODS}

This was a retrospective descriptive study where survivors records were examined. Sources of records included hospitals, health facilities and rehabilitation centres at which survivors attended, Africa Medical Research Foundation (AMREF) and at the author's follow up clinic. The study period was 1999 to2002.

\section{RESULTS}

Three hundred and fifteen survivors had orthopaedic conditions. Out of $305,64 \%$ were married, $22 \%$ were single, while $64 \%$ had between $0-3$ and $25 \%$ had $4-6$ children. Of the 277 followed up survivors, females comprised (172) (62.1\%). F:M ratio 1.6:1.

At the time of the blast $71.8 \%$ of the victims were inside buildings and $75.1 \%$ were within 100 metres radius from the blast site. Health facility first attended: Public hospitals $50.0 \%$, Private hospitals $33.2 \%$ and health clinics $16.8 \%$. 


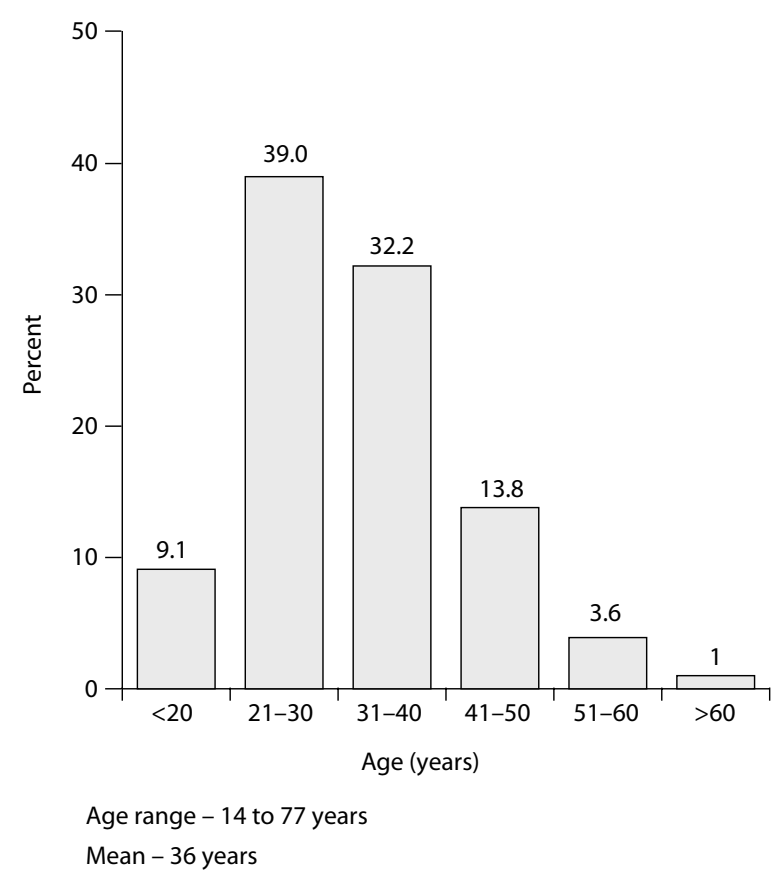

Figure 1: Age distribution of 277 patients with orthopaedic conditions

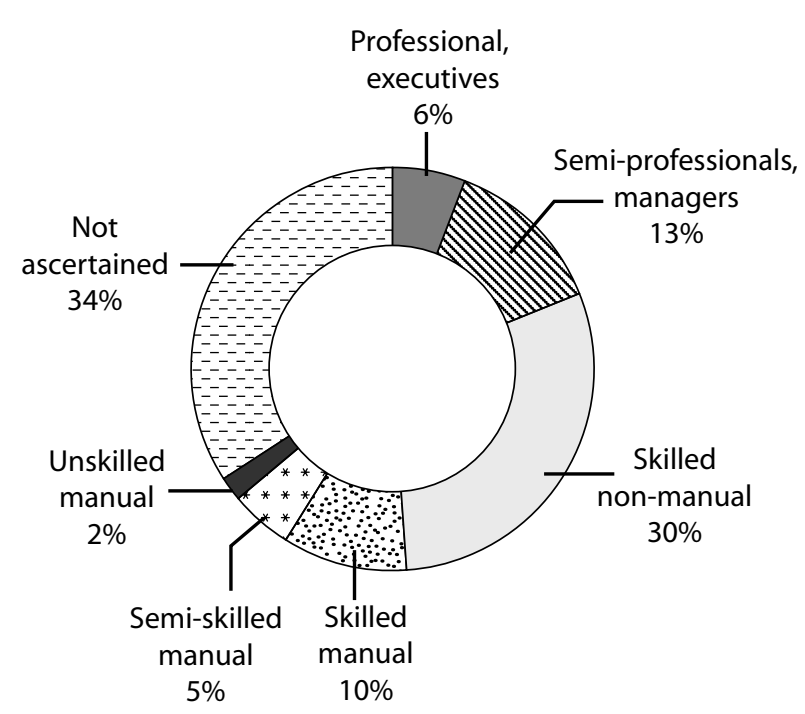

Figure 2: Occupation

Table 1

Location and radius versus injuries in 136 survivors

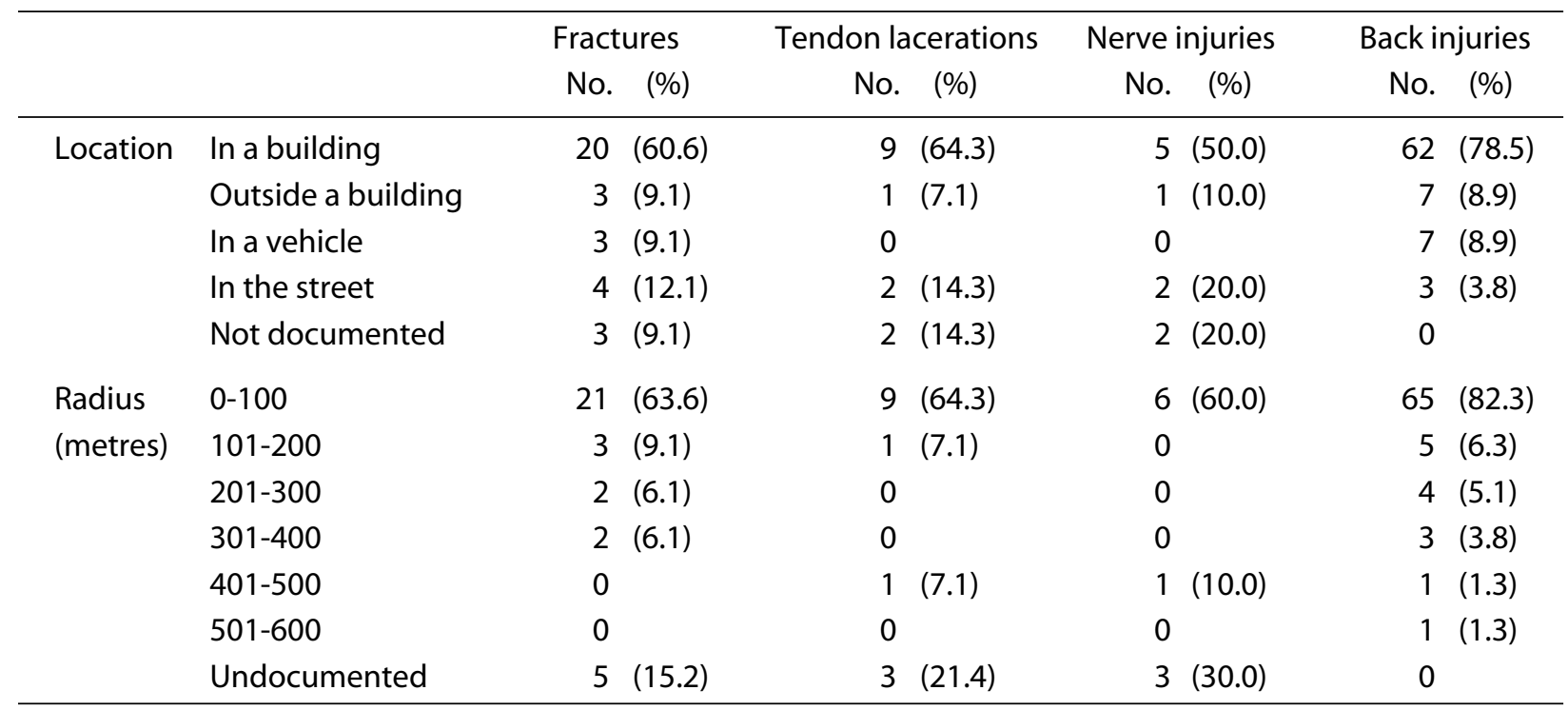

Inside buildings $70.6 \%$ and within 100 metres radius $74.3 \%$. 
Table 2

Joint injuries and associated fractures or dislocation - Percentage in brackets

\begin{tabular}{|c|c|c|c|}
\hline Joint & $\begin{array}{l}\text { Without fracture } \\
\text { No. (\%) }\end{array}$ & $\begin{array}{l}\text { With associated fracture } \\
\text { No. (\%) }\end{array}$ & $\begin{array}{l}\text { With associated dislocation } \\
\text { No. (\%) }\end{array}$ \\
\hline Shoulder & $30(37.0)$ & 0 & $2(40.0)$ \\
\hline Knee & $18(22.0)$ & $1 \quad(16.7)$ & 0 \\
\hline Ankle & $18(22.0)$ & $3 \quad(50.0)$ & 0 \\
\hline Hip & $5 \quad(6.2)$ & 0 & 0 \\
\hline Elbow & $2 \quad(2.5)$ & 0 & 0 \\
\hline Wrist & $4 \quad(4.9)$ & $1 \quad(16.7)$ & 0 \\
\hline Foot & $1 \quad(1.2)$ & $1 \quad(16.7)$ & 0 \\
\hline Finger/thumb & $3 \quad(3.7)$ & 0 & $1(20.0)$ \\
\hline Patellofemoral & 0 & 0 & $1(20.0)$ \\
\hline Sternoclavicular & 0 & 0 & $1(20.00)$ \\
\hline Total & $81(100)$ & $6 \quad(100)$ & $5(100)$ \\
\hline
\end{tabular}

(No. $=67)$

Table 3

Location of post/non - traumatic conditions

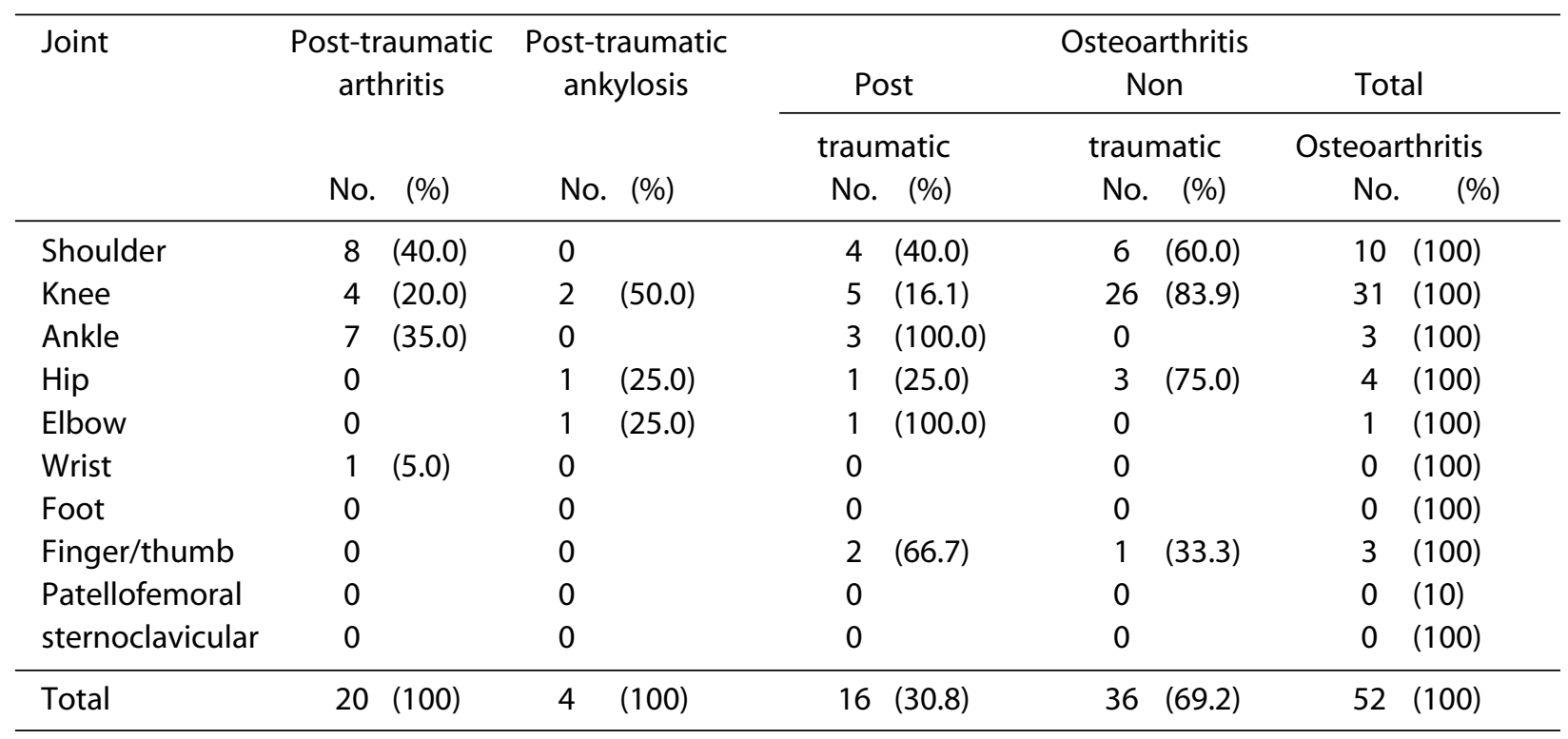

Table 4

Back injuries

\begin{tabular}{|c|c|c|c|c|c|c|c|c|c|c|}
\hline & \multicolumn{2}{|c|}{ Level of back injury } & \multicolumn{4}{|c|}{ Associated spondylosis } & \multicolumn{4}{|c|}{ Associated proplased Invertebral disc } \\
\hline & \multirow[b]{2}{*}{ No. } & \multirow[b]{2}{*}{$(\%)$} & \multicolumn{2}{|c|}{ Traumatic } & \multicolumn{2}{|c|}{ Non Traumatic } & \multicolumn{2}{|c|}{ Traumatic } & \multicolumn{2}{|c|}{ Non-traumatic } \\
\hline & & & No. & $(\%)$ & No. & $(\%)$ & No. & $(\%)$ & No. & $(\%)$ \\
\hline Cervical & 6 & $(6.8)$ & 0 & & 13 & $(15.7)$ & 0 & & 0 & \\
\hline Thoracic & 7 & $(8.0)$ & 1 & $(1.2)$ & 4 & $(4.8)$ & 0 & & 0 & \\
\hline Lumbar & 75 & $(85.2)$ & 2 & (3.1) & 163 & $(75.9)$ & 3 & $(50.0)$ & 3 & $(50.0)$ \\
\hline Total & 88 & $(100)$ & 3 & (3.6) & 80 & $(96.4)$ & 3 & $(50.0)$ & 3 & $(50.0)$ \\
\hline
\end{tabular}


Table 5

Operations performed

\begin{tabular}{lrr}
\hline Operation & No. & $(\%)$ \\
\hline Internal fixation of bone & 10 & 19.6 \\
External fixation & 1 & 2.0 \\
Arthrodesis (fusion) & 2 & 3.9 \\
Tendon repair & 8 & 15.7 \\
Osteotomy & 1 & 2.0 \\
Removal of foreign bodies & 11 & 21.6 \\
Removal of plate & 4 & 7.8 \\
Excision of ectopic bone & 3 & 5.9 \\
Patellectomy & 1 & 2.0 \\
Laminectomy & 2 & 3.9 \\
Amputation & 3 & 5.9 \\
Sequestrectomy / debridement/ drainage & 3 & 5.9 \\
Partial resection in growing toe nail & 1 & 2.0 \\
Excision of neuroma & 1 & 2.0 \\
\hline Total & 51 & 100 \\
\hline
\end{tabular}

The following 64 complications were noted:Post-traumatic arthritis 20 (31.3\%), Post-traumatic osteoarthritis 16 (25.0\%), Post-traumatic ankylosis and tenosynovitis 4 (6.3\%) each, joint stiffness, prolapsed intervertebral disc, spondylosis $3(4.7 \%)$ each, post-traumatic ossification $2(3.1 \%)$ and recurrent shoulder dislocation and patella, chronic osteomyelitis, metacarpal malunion, non-unison, ingrowing toe nail, abscess, post-traumatic neuroma and fracture ribs with pneumothorax one (1.6\%) each.

\section{DISCUSSION}

Out of a study population of 1090, $315(29 \%)$ had orthopaedic conditions. Of the 305 documented cases $64 \%$ were married and $32 \%$ single while $64 \%$ had between 0 to 3 and $25 \%$ had 4 to 6 children. No similar study was found. A number of the victims may have been unable to return to their previous occupations there by being unable to cater for their dependants. Two hundred and seventy seven survivors were followed up and $86 \%$ were between 21 to 50 years. This compares with the Oklahoma City bombing in which over $80 \%$ were between ages $20-59$ year. The bomb victims were in the economically important group. The age range in the study was 14 to 77 years with a mean of 36 years.

Overall, females comprised $63.1 \%$ of the victims compared to $37.9 \%$ males, giving female to male ratio of 1.6:1. Hadden and his colleagues found a similar overall ratio (1). During the world trade centre attack in New York, there were more female victims (66.1\%), which compares with the above findings (8). However, the female to male ratio was reversed (1:1.4) when considering those who were admitted to hospital in the study done by Hadden et al (1).

When considering the locations at the time of the blast, $71.8 \%$ were inside buildings while $8.7 \%$ were outside and $75.5 \%$ were within 100 metres from the blast site. It is therefore evident that being inside a building that was close to the bomb blast site carried a higher risk of injury as evidenced in this study.

The study on the Oklahoma bombing showed a similar trend. When considering only the 675 nonfatal injuries, $412(61 \%)$, occurred in victims inside buildings that were in close proximity to the bomb explosion site (7). The majority of the victims in this study were economically productive; professionals, executives, semiprofessionals, managers, skilled nonmanual and skilled manual workers comprised $89.5 \%$, when considering only those whose occupations were documented, but when the undocumented survivors were included they comprised 59\%. Semi-skilled and unskilled manual workers made up only $10.5 \%$ of the victims. A comparable study was not found.

Fifty per cent of the victims were treated in public hospitals while private hospitals and health clinics attended to $33.2 \%$ and $16.8 \%$ of the victims respectively. As the majority the victims were treated in public hospitals, this underscores the need to adequately fund, equip and staff government hospitals. In the Oklahoma bombing, out of 593 victims who were treated and 
released, 359 (60.4\%) were treated in area hospitals and emergency rooms while 233 (39.4\%) were treated in private physicians offices. During the Oklahoma City bombing, $57(13.9 \%)$ of the 442 victims treated in area hospitals sustained fractures or dislocations (7). This is similar (11.9\%) to the current study.

Among the 790 survivors of the terrorist attack on the World Trade Centre in New York, only 46 (6\%) sustained fractures (8). Hadden and colleagues had a fracture rate of $3.5 \%$ among 1523 bombing victims (1). The majority of the fracture victims were inside buildings and within 100 metres from the bomb blast site.

The victims were injured when they fell due to the bomb blast, or while trying to escape or object or objects fell on them.

Out of the 277 victims who were followed up, $67(24.3 \%)$ sustained, 92 joint injuries. Eighty one joints (88.0\%) had no associated fractures while six joints had associated fractures of which three involved ankle joint while five joints had associated dislocations. The joint injuries involved the shoulder in 30 cases (37.0\%), the ankle and the knee in 18 cases each $(22.2 \%)$. The victims who were inside buildings at the time of the blast comprised $82.1 \%$ while $79.1 \%$ were within radius of 100 meters. No comparable study of joint injuries was found. Post-traumatic arthritis occurred in 30 (31.7\%) joints. The shoulder or ankle joint was involved in 15 (75\%) cases.

A total of 52 joints had osteoarthritis of which $16(30.8 \%)$ were due to joint trauma, the knee joint accounting for 31(59.6\%). Ankylosis occurred in four, (4.3\%) joints. No comparable studies were found.

Hadden et al reported six tendon lacerations that were repaired out of 1523 survivors (1), while in Oklahoma bombing, no specific mention of tendon injuries among the survivors who sustained lacerations, abrasions and contusions was made (7).

Nerve injuries were $10(3.6 \%)$ of which a half sustained injuries inside buildings and six of them were within 100 metres from the blast site. Laceration of the branch of the superficial radial nerve occurred in one victim. They were injured by flying objects, including shattered glass. Hadden et al recorded six major nerve damages, which included one sciatic, two radial and one median nerves (1).

Back injuries occurred in 79 victims $(28.5 \%)$. The lumbar spine accounted for 75 (85.2\%) of the 88 levels and this was statistically significant. The victims who were inside buildings comprised $78.5 \%$ while $82.3 \%$ were within 100 metres from the blast site. Many were injured when they fell due to the blast, or when trying to escape or objects fell on them. Spondylosis was noted at 83 levels out of which three (3.6\%) were as a result of trauma since they were young enough not to have it. Prolapsed intervertebral disc occurred in six victims and only three were attributable to trauma. Three cases of non-trauma related spondylolithesis were noted. No comparable study was found.

A total of 51 operations were performed on victims who were under orthopaedic care. Internal fixation of fractures, tendon repair and removal of foreign bodies accounted for $56.9 \%$ of the operations. No details of operations were documented in other studies $(1,3,7,8)$. A total of 64 complications were noted with posttraumatic arthritis and osteoarthritis accounting for 36 (56.3\%).

The economic impact of the bomb blast that occurred at the American Embassy in Nairobi, Kenya on the $7^{\text {th }}$ August 1998 has not been fully assessed, but must be considerable, when damaged property, loss of human life, loss of earnings due to the inability to continue working and the cost of treatment is put into account. In the Oklahoma City bombing, the charges for acute care at hospitals exceeded 2.5 million U.S dollars. This excluded emergency transport, doctors and rehabilitation charges. When these are considered together with the damage to property, the overall cost would be considerable (7).

\section{CONCLUSION AND RECOMMENDATIONS}

The bomb blast victims were in economically productive group with the majority having 0-3 dependants. Being inside a building and within 100 metres from the blast carried the highest risk of injury. Fifty percent of the survivors, received their first treatment in public hospitals. The economic impact when fully assessed will be considerable.

The following recommendations were suggested:-

(i) Formation of disaster management body that will draw up protocols for handling disasters.

(ii) Basic first aid education should be instituted in schools/colleges, work places and the civil society.

(iii) Advanced trauma life support training for medical personnel should be enhanced.

(iv) Adequate funding, expansion and modernization of the fire departments in all towns. 
(v) Retraining and instilling proper work ethics among fire fighters.

(vi) Ambulance services should be introduced in all urban centres by the Government, private sector and charitable organizations.

(vii) Buildings by laws should be re-examined with a view of improving standards and making buildings safer.

(viii) Adequate funding, staffing and equipping of public hospitals, should be reviewed as the institutions handled the bulk of bomb blast victims.

(ix) Measures should not be spared to improve the efficiency of our security apparatus to prevent occurrence of such attacks in future.

\section{REFERENCES}

1. Hadden W.A., Rutherford W.H. and Merret J. A. The injuries of terrorist bombing. A study of 1532 consecutive patients. Brit. J. Surg. 1978; 65: 525-531.
2. Severance H.W. The blast-related injuries. Special considerations for mass casualties management. Traumavue. 1999; 7: No. 1, Spring.

3. Karmy-Jones R., Kissinger D., Golocovsky M., Jordan M. and Champion H.R. Bomb related injuries. Mil. Med. 1994; 159: 536-539.

4. Part I: Types of wounds and injuries, Chapter V. Blast injuries emergency war surgery. NATO Handbook.

5. Hull J.B., Bowyer G.W., Cooper G.J. and Crane J. Pattern of injury in those dying from traumatic amputation caused by bomb blast. Brit. J. Surg. 1994; 81: 1132-1135.

6. Hull J.B. and Cooper G.J. Pattern and mechanism of traumatic amputation by explosive blast. J. Trauma Injury Infec. Critical Care 1996; 40: 1985-2055.

7. Injury prevention services, Oklahoma State Department of Health. Investigations of physical injuries directly associated with Oklahoma City Bombing

8. New York City Dept. of Health. Rapid assessment of Injuries on the World Trade Center-New York City. Sept. 2001. Morbidity and Mortality Weekly Report. 2002; vol. 51. 